\title{
Enterprise risk management in financial groups: analysis of risk concentration and default risk
}

\author{
Nadine Gatzert • Hato Schmeiser • \\ Stefan Schuckmann
}

Published online: 2 July 2008

(C) Swiss Society for Financial Market Research 2008

\begin{abstract}
In financial groups, enterprise risk management is becoming increasingly important in controlling and managing the different independent legal entities in the group. The aim of this paper is to assess and relate risk concentration and joint default probabilities of the group's legal entities in order to achieve a more comprehensive picture of a financial group's risk situation. We further examine the impact of the type of dependence structure on results by comparing linear and nonlinear dependencies using different copula concepts under certain distributional assumptions. Our results show that even if financial groups with different dependence structures do have the same risk concentration factor, joint default probabilities of different sets of subsidiaries can vary tremendously.
\end{abstract}

Keywords Enterprise risk management · Copulas · Financial groups · Joint default risk

JEL Classification G20 · G28 · C16

\section{Introduction}

During the last several years, there has been a trend toward consolidation (M\&A activities) in the financial sector of many countries (see Amel et al. 2004). In the course

\footnotetext{
N. Gatzert · H. Schmeiser $(\bowtie) \cdot$ S. Schuckmann

University of St. Gallen, Institute of Insurance Economics, Kirchlistrasse 2, 9010 St. Gallen,

Switzerland

e-mail: hato.schmeiser@unisg.ch

N. Gatzert

e-mail: nadine.gatzert@unisg.ch

S. Schuckmann

e-mail: stefan.schuckmann@unisg.ch
} 
of this consolidation, large financial groups have been formed that provide financial services in different sectors and countries. In the context of insurance markets, the European Union (EU) distinguishes between financial conglomerates and insurance groups. A financial group providing services and products in different sectors of financial markets is called a "financial conglomerate" (see Art. 14 of European Council 2002). "Insurance groups" are defined as financial groups providing insurance services, but not necessarily providing services in other sectors (see Art. 1 of European Council 1998). In this article, we analyze the more general structure of a financial group that comprises insurance groups and financial conglomerates.

Regulatory authorities, as well as rating agencies, are concerned with new types of risk and risk concentrations arising in these groups and how to properly assess them in group supervision. The Solvency II project within the European Unionespecially the quantitative impact studies (QIS) — will intensify discussion and make more urgent the need for practicable implementations (for the discussion on group solvency, see, e.g., CEIOPS 2006; on QIS, see, e.g., CEIOPS 2007). In this context, enterprise risk management (ERM) has become increasingly important. ERM takes a comprehensive view of risk and helps manage risks in a holistic and consistent way (CAS 2003). The aim of this paper is to provide a detailed and more comprehensive picture of an insurance group's risk situation by assessing and relating the risk concentration and joint default probabilities of its legal entities. We further examine the impact of the type of dependence structure on results by comparing linear and nonlinear dependencies using the concept of copulas.

Determination of the risk concentrations of an insurance group can be based on an analysis of diversification effects at a corporate level since diversification is the opposite of concentration. In particular, risk concentrations, like interdependencies or accumulation, reduce diversification effects. The diversification effect is measured with the economic capital of an aggregated risk portfolio, which implicitly relies on the assumption that different legal entities are merged into one. An essential aspect in aggregating risks is modeling the dependence structure using linear and nonlinear dependencies. Copula theory can be used to model nonlinear dependencies in extreme events and to test the financial stability of a group structure.

There has been steady growth in research on the application of copula theory to risk management. Embrechts et al. (2002) introduce copulas in finance theory and analyze the effect of dependence structures on value at risk. Li (2000) applies copulas to the valuation of credit derivatives. Frey and McNeil (2001) model dependent defaults in credit portfolios, with a special emphasis on tail dependencies. An introduction to the use of copulas in the actuarial context can be found in Frees and Valdez (1998) and Embrechts et al. (2003).

A central aspect of ERM is the aggregation of different types of risk to calculate the economic capital necessary as a buffer against adverse outcomes. Wang (1998, 2002) gives an overview on the theoretical background of economic capital modeling, risk aggregation, and the use of copula theory in enterprise risk management. Kuritzkes et al. (2003) aggregate risks at different levels of a financial holding company under the assumption of joint normality; in an empirical study, they compute the relative diversification effect for several conglomerates. Ward and Lee (2002) use a normal copula approach to aggregate the risks of a diversified insurer in a combined 
analytical and simulation model. Dimakos and Aas (2004) apply a similar method to model total economic capital, and combine risks by pairwise aggregation; they present a practical approach to estimate the joint loss distribution of a Norwegian bank and a Norwegian life insurance company.

Faivre (2003) models the overall loss distribution for a four-lines-of-business insurance company and examines the influence of different types of copulas on the value at risk and the company's default probability. Tang and Valdez (2006) simulate the economic capital requirements for a multiline insurer, taking into account different types of distributions and different types of copulas; the resulting values for economic capital are used to compute absolute diversification benefits. Rosenberg and Schuermann (2006) relax the joint normality assumption and use copula theory to aggregate risks with nonnormal marginals; they analyze the influence of the business mix between credit, market, and operational risk on value at risk and calculate diversification benefits by comparing the value at risk of the diversified conglomerate with the stand-alone value at risk.

However, most of the literature does not take into account the special risk profile of financial groups that arises from the group-holding structure. In particular, rigorous legal restrictions from, e.g., insurance law, company law, and insolvency law apply to intragroup transfers and may prevent loss compensation between different legal entities in a group. In the European Union, for example, combining banking and insurance activities in the same legal entity is prohibited (see Article 6(1b) of the Life Insurance Directive 2002/83/EC and Article 8(1b) of the Nonlife Insurance Directive 73/239/EEC). Article 18(1) of the Life Insurance Directive prohibits the combination of life and nonlife business in the same legal entity. As these legal frameworks are rather focused on the stability of an individual firm, they may get in the way of group interests with respect to transfers of funds in case of financial distress. Hence, where such is even lawful, a transfer of funds between different legal entities in case of an insolvency of one entity occurs only if a transfer-of-losses contract has been signed or if the management of the corporate group decides in favor of cross-subsidization (e.g., for reputational reasons).

Since the contracting party is usually not the whole group, but merely a single subsidiary, in principle, the structure of an insurance group is not important to those buying insurance from the subsidiary in respect to the insurer's default risk. Thus, generally, for policyholders and other debt holders, only the default risk of individual legal entities and their ability to meet outstanding liabilities are of relevance when there is no transfer-of-losses contract between members of the group. However, for the executive board of the insurance group and for shareholders, information on diversification - and thus on risk concentration — and joint default probabilities is important when considering the risk profile of the group for ERM. Information on risk concentration may also be helpful in obtaining a certain rating level from a rating agency. From the perspective of regulatory agencies, risk concentration information can be valuable in analyzing systemic risk of insolvency. The default of a whole financial group will, in general, have a stronger impact on financial markets than the default of a single subsidiary company.

This paper extends the literature described above by analyzing risk concentrations in an insurance group and by concurrently reporting joint default probabilities for sets 
of legal entities within a financial group. Noting that joint default probabilities only depend on individual default probabilities and the coupling dependence structure, we further study the influence of different dependence structures using the concept of copulas. In particular, we consider an insurance group with three legal entities and compare results for Gauss, Gumbel, and Clayton copulas for normal and nonnormal marginal distributions.

Our results demonstrate that even if different dependence structures imply the same risk concentration factor for the financial group, joint default probabilities of different sets of subsidiaries can vary tremendously with the dependence structure. The analysis shows that the simultaneous consideration of risk concentration and default probabilities can provide information of substantial value.

The remainder of the paper is organized as follows. Section 2 introduces the concept of diversification on economic capital and risk concentration of a financial group. Furthermore, dependence structures are presented, including linear and nonlinear dependencies modeled with copulas. In the numerical analysis, Sect. 3, we compare results for Gauss, Gumbel, and Clayton copulas under different distributional assumptions. Section 4 concludes.

\section{Risk concentration, default risk, and dependence structures}

This section describes, first, a framework for measuring risk concentrations by calculating the diversification effect on the economic capital of an insurance group, assuming that the different legal entities are merged. The economic capital is the amount necessary to buffer against unexpected losses from business activities so as to prevent default at a specific risk tolerance level for a fixed time horizon. Diversification is generally intended to reduce the overall risk level in an insurance group, and thus acts to alleviate the dangers inherent in risk concentration. Calculating risk concentration in an insurance group can thus be based on an examination of diversification effects at the group level. In a second step, the joint default probabilities of legal entities within a group are introduced. Third, linear and nonlinear dependence structures are discussed.

We focus on an insurance group with a holding structure and different companies (legal entities) with limited liability. Generally, such a group is subject to market risks, credit risks, underwriting risks, and operational risks. If each member of the group is faced with similar risks, one would expect the stochastic liabilities of the different entities to be highly correlated. This will usually be the case if several firms of the same type, for example, financial firms, form a group. However, if the corporate group is composed of companies from widely different industries, the liabilities between the different legal entities might be rather uncorrelated. In what follows, we will consider an insurance group consisting of a bank, a life insurer, and a nonlife insurer.

In our framework, the equity value of each subsidiary legal entity is modeled at two points in time $(t=0,1)$. The value of the assets (liabilities) at time $t=1$ of company $i$ is defined as $A_{i}\left(L_{i}\right)$. Debt and equity capital in $t=0$ is invested in riskless assets, leading to a deterministic cash flow for the assets in $t=1$, whereas liabilities paid out in $t=1$ are modeled stochastically. 


\subsection{Stand-alone economic capital}

The amount of necessary economic capital depends on the specific risk tolerance level and on the measure chosen to evaluate corporate risk. In the following, we determine the necessary amount of capital using the default probability. The default probability $\alpha$ of each legal entity $i$ can be written as $P\left(A_{i}<L_{i}\right)=\alpha_{i}$.

In the next step, the invested assets $A_{i}$ are divided into two parts-the expected value of the liabilities $E\left(L_{i}\right)$ and the economic capital $E C_{i}$ leading to $P\left(L_{i}-E\left(L_{i}\right)>E C_{i}\right)=\alpha_{i}$. Hence, given a probability distribution for the liabilities and a certain safety level $\alpha_{i}$, the economic capital $E C_{i}$ can be derived. The necessary economic capital $E C_{i}$ for $N$ different legal entities within a financial group can be calculated by

$$
E C_{i}=\operatorname{VaR}_{1-\alpha}\left(L_{i}\right)-E\left(L_{i}\right), \quad i=1, \ldots, N .
$$

For consistency, all companies within the group should have the same safety level $\alpha$. Therefore, $\operatorname{VaR}$ is defined by $\operatorname{VaR}_{\alpha}\left(L_{i}\right)=F_{L_{i}}^{-1}(\alpha)=\inf \left\{x \mid F_{L_{i}}(x) \geq \alpha\right\}$, where $F_{L_{i}}$ stands for the distribution function of the liabilities for company $i$.

\subsection{Aggregated economic capital}

Assuming that the several companies in the group are merged into one company (full liability between legal entities), the necessary economic capital for the safety level $\alpha$ on an aggregate level for $\sum_{i=1}^{N} L_{i}$ can be written as

$$
E C_{a g g r}=V a R_{1-\alpha}\left(\sum_{i=1}^{N} L_{i}\right)-E\left(\sum_{i=1}^{N} L_{i}\right) .
$$

To calculate the quantile in (2), information about the cumulative distribution of the liabilities is needed. Closed-form solutions for $\sum_{i=1}^{N} L_{i}$ can be derived only for a limited number of distributions. In the case of a normal distribution, only the variance of the portfolio is needed to determine the aggregate economic capital $E C_{a g g r}$. If no closed-form solution can be obtained, the quantile of the distribution of the aggregate liabilities $\sum_{i=1}^{N} L_{i}$ can be estimated using either numerical simulation techniques or analytical approximations (for an overview, see Daykin et al. 1994, pp. 119 ff; Ammann and Reich 2001).

\subsection{Diversification versus concentration}

Given (1) and (2), diversification can be measured with the ratio of aggregated economic capital to the sum of stand-alone economic capital (see, e.g., Kuritzkes et al. 2003),

$$
d=\frac{E C_{a g g r}}{\sum_{i=1}^{N} E C_{i}} .
$$

In the case of linear dependencies, the factor $d$ takes on values between zero and one and can be used to compare the level of risk concentration in groups. A value of one 
corresponds to perfect correlation, which means that there would be no diversification benefits if the different legal entities merged into one company. When risk factors are less than perfectly correlated, some of the risk can be diversified. Given a benchmark company, a higher value of $d$ implies possible risk concentration, since lower values of $d$ mean a higher diversification and thus a lower risk concentration. We henceforth refer to the coefficient in (3) as the risk concentration factor. To keep the different quantities in (3) comparable, it is important to use the same risk measure and the same time horizon for all legal entities when calculating the economic capital.

Other ways of deriving the concentration factor $d$ include absolute measures for diversification, which have been used in the literature (see, e.g., Tang and Valdez 2006). Absolute measures reveal the actual loss a group faces, but they do not allow comparison of companies and groups that are of different sizes. Furthermore, one can change the risk measure for calculating stand-alone and aggregated economic capital by using, e.g., the expected shortfall or expected policyholder deficit instead of $V a R$. These risk measures have the property of being coherent and, in addition, account for losses in the tail of the distribution. In the present setting, however, VaR was chosen because of its planned integration in the Solvency II framework in the European Union at a confidence level of $99.5 \%$ (see Art. 100 of the proposal in European Commission 2007), thus being of high practical relevance despite its shortcomings.

Generally, diversification of the group is of no relevance to debt holders of the group's individual companies (e.g., policyholders) since the whole group is not the contracting party, that is, the contract is between the policyholder and the insurance subsidiary only (although there might be transfer-of-loss contracts). However, for management and shareholders of the corporate group, information about risk concentration in the different sectors is of high importance.

\subsection{Determination of default probabilities}

Even though calculation of the diversification factor may enable the detection of risk concentrations within the group, the factor is in most cases only a hypothetical number since individual legal entities generally do not (fully) cover the losses of the other entities. To obtain further insight about the group's risk situation, joint default probabilities are appropriate and can provide additional and valuable information.

In contrast to the determination of the risk concentration factor, which requires a convolution over different entities $\sum_{i=1}^{N} L_{i}$, default probabilities make use of only the joint distribution function. To determine the joint default probability of two or more legal entities, the joint cumulative distribution function is needed. ${ }^{1}$ For the case of a group comprised of three legal entities, the joint default probabilities of exactly one, two, and three legal entities are given by

\footnotetext{
${ }^{1}$ For example, for the case of a group comprised of three legal entities, the joint default probabilities of exactly one, two, and three legal entities are given by

$$
\begin{aligned}
& P_{1}=1-P\left(L_{1} \leq A_{1}, L_{2} \leq A_{2}, L_{3} \leq A_{3}\right)-P_{2}-P_{3}, \\
& P_{2}=P\left(L_{i}>A_{i}, L_{j}>A_{j}, L_{k} \leq A_{k}, i \neq j \neq k\right), \\
& P_{3}=P\left(L_{1}>A_{1}, L_{2}>A_{2}, L_{3}>A_{3}\right) .
\end{aligned}
$$
}

It is assumed that no transfer of losses between companies will occur. 


\subsection{Modeling the dependence structure}

In risk management, appropriate modeling of dependence structures is very important. One recommendation in the literature is to apply copulas in addition to linear correlation to ensure an adequate mapping of dependence (see Embrechts et al. 2002). Copulas allow for the inclusion of features such as fat tails and skewness for nonelliptically distributed risks, which are generally not captured with standard multivariate distributions. For continuous multivariate distribution functions, copulas represent the multivariate dependence structure and couple the univariate marginal distributions to a joint multivariate distribution (see Nelsen 1999).

Hence, the joint default probability depends on the dependence structure expressed by the copula and on the marginal default probabilities. In our case, these quantities are given and fixed since the economic capital for each entity is adjusted such that the marginal default probabilities remain constant. In the analysis, we will compare several copulas. To obtain boundaries, we include the case of independence and perfect dependence (comonotonicity) and the independence copula (see McNeil et al. 2005, p. 189). Furthermore, the two most common Archimedian copulas, Clayton and Gumbel, are used to model the dependence structure between the entities. These are explicit copulas that have closed-form solutions and are not derived from multivariate distribution functions as is the implicit Gaussian copula. Both Clayton and Gumbel copulas exhibit asymmetries in the dependence structure. The Clayton copula is lower tail dependent; the Gumbel copula is upper tail dependent.

Linear dependence is a special case of the copula concept. Given a multivariate Gaussian random vector, then its copula is a so-called Gauss copula (McNeil et al. 2005, p. 191). The Gauss copula measures the degree of monotonic dependence and has no closed-form solution, only an integral representation. ${ }^{2}$ If the joint distribution is a multivariate normal with standard normal marginals, the economic capital $E C_{i}$ for each entity can be calculated by (see, e.g., Hull 2003, pp. 350 ff.)

$$
E C_{i}=\sigma\left(L_{i}\right) \cdot z_{\alpha},
$$

where $z_{\alpha}$ denotes the $\alpha$-quantile of the standard normal distribution and $\sigma$ stands for the standard deviation. To aggregate the economic capital under the assumption that all sectors are carried in one company, correlations coefficients $\rho_{i j}$ between the liabilities of entity $i$ and entity $j$ are needed. Given the standard deviation of the portfolio of the liabilities $L=\sum_{i=1}^{N} L_{i}$, the aggregated economic capital-assuming that all sectors in the financial group are merged - can be calculated from (see Kuritzkes et al. 2003; Groupe Consultatif 2005):

$$
E C_{a g g r}=\sigma(L) \cdot z_{\alpha}=\sqrt{\left(\begin{array}{c}
E C_{1} \\
E C_{2} \\
\vdots \\
E C_{N}
\end{array}\right)\left(\begin{array}{cccc}
1 & \rho_{12} & \cdots & \rho_{1 N} \\
\rho_{21} & 1 & \cdots & \rho_{2 N} \\
\vdots & \vdots & \ddots & \vdots \\
\rho_{N 1} & \rho_{N 2} & \cdots & 1
\end{array}\right)\left(\begin{array}{c}
E C_{1} \\
E C_{2} \\
\vdots \\
E C_{N}
\end{array}\right) .}
$$

\footnotetext{
${ }^{2}$ The copula may be constructed by the inverse method, which maps linear dependence in the form of the linear correlation of ranks, as described by Iman and Conover (1982) and by Embrechts et al. (2002).
} 
Equation (5) illustrates that the effect of diversification on the aggregated economic capital $E C_{a g g r}$ depends on the number $N$ of legal entities, the relative portion of the economic capital of the individual companies $E C_{i}$, and the correlation between the liabilities of the different companies.

\section{Simulation analysis}

In this section, we present numerical examples in order to examine the influence of the dependence structure (nonlinear vs. linear dependence) and the distributional assumptions (normal vs. non-normal) on risk concentration and default probabilities. First, the case of linear dependence is presented for normally and nonnormally distributed liabilities with different sizes. Second, nonlinear dependencies are examined for normality and nonnormality. Table 1 sets out the input parameters that are the basis for the numerical examples analyzed in this section. The values and distributions in this simulation study are chosen to demonstrate fundamental effects of nonlinear dependencies on the risk situation of a financial group and should help increase companies' awareness with respect to nonlinear dependencies.

Table 1 contains values for two different cases, (A) and (B), for normally and nonnormally distributed liabilities. The given default probability of $0.50 \%$ is adapted to Solvency II regulatory requirements, which are currently being debated (European Commission 2005). For normally distributed liabilities, economic capital can be calculated using (4) with a standard normal quantile of $z_{\alpha}=2.5758$. The group under consideration consists of a bank, a life insurance company, and a nonlife insurer. In case (A), the liabilities of all three entities have the same standard deviation, and thus require the same economic capital. In case (B), the bank has a substantially higher standard deviation than the insurance entities. Accordingly, the resulting economic capital differs.

We next change the distribution assumption to allow for nonnormality. Now, only the liabilities of company 1 (Bank) are normally distributed, whereas the liabilities

Table 1 Economic capital for individual entities in a financial group for different distributional assumptions given a default probability $\alpha=0.50 \%$ and $E\left(L_{i}\right)=100, i=1,2,3$

\begin{tabular}{|c|c|c|c|c|c|}
\hline \multirow[t]{2}{*}{ Legal entity } & \multirow[t]{2}{*}{ Distribution type } & \multicolumn{2}{|c|}{ Case (A) } & \multicolumn{2}{|c|}{ Case (B) } \\
\hline & & $\overline{\sigma\left(L_{i}\right)}$ & $E C_{i}$ & $\overline{\sigma\left(L_{i}\right)}$ & $E C_{i}$ \\
\hline & "normal" & & & & \\
\hline Bank & Normal & 15.00 & 38.64 & 35.00 & 90.15 \\
\hline Life insurer & Normal & 15.00 & 38.64 & 5.00 & 12.88 \\
\hline Nonlife insurer & Normal & 15.00 & 38.64 & 5.00 & 12.88 \\
\hline \multirow[t]{2}{*}{ Sum } & & & 115.91 & & 115.91 \\
\hline & "non-normal" & & & & \\
\hline Bank & Normal & 15.00 & 38.64 & 35.00 & 90.15 \\
\hline Life insurer & Lognormal & 15.00 & 45.22 & 5.00 & 13.59 \\
\hline Non-life insurer & Gamma & 15.00 & 42.84 & 5.00 & 13.35 \\
\hline Sum & & & 126.70 & & 117.09 \\
\hline
\end{tabular}


of company 2 (Life Insurer) and company 3 (Nonlife Insurer) follow, respectively, a lognormal and a gamma distribution. To keep the cases comparable, the expected value $\mu$ and standard deviation $\sigma$ remain fixed. ${ }^{3}$

The assumption of nonnormal distributions leads to different individual economic capital values in case (A) and case (B) compared to the values under the normality assumption. As a result, the sum of the individual economic capital (126.70 in case (A) and 117.09 in case (B)) differs also (115.91 for both cases under the normality assumption).

The numerical analysis proceeds as follows. First, we calculate the necessary aggregated economic capital based on the value at risk at the group level for a confidence level $\alpha=0.50 \%$ (see (2)). ${ }^{4}$ The concentration factor can then be derived using the stand-alone economic capital of the legal entities given in Table 1 by way of (3). Subsequently, we calculate the corresponding default probabilities $P_{1}, P_{2}, P_{3}$ (i.e., the probability that exactly one, two, or three entities default).

\subsection{Numerical results for linear dependence}

To calculate the necessary economic capital at the group level, the correlation matrix for the liabilities is needed. Estimation of dependencies can be made on the basis of macroeconomic models. For instance, Estrella (2001) derives linear correlation factors from US stock market returns to measure possible diversification effects for assumed mergers in the banking and insurance sectors. In Bikker and Lelyveld (2002), correlation factors between bank and insurance sectors of different countries are estimated, based on financial market data, to evaluate possible cross-sector mergers.

To obtain more comprehensive information on the risk situation of group under consideration (see Table 1), we compare the effect of distributional assumptions on the concentration factor and default probabilities. Figure 1 shows a plot of the default probabilities for different choices of the correlation matrix with increasing dependency and the corresponding concentration factors for different distributional assumptions. In particular, we compare the cases (A) and (B) given in Table 1 when liabilities follow a normal distribution (normal) and when they are partly nonnormally distributed (nonnormal). For ease of exposition, we use the same coefficient of correlation $\rho$ between the liabilities of all entities, i.e., $\rho\left(L_{i}, L_{j}\right)=\rho, i \neq j$.

Figure 1 shows how the concentration factor and information on default probabilities can complement each other. Part (a) illustrates that the joint default probabilities depend on the dependence structure between the legal entities and individual default probabilities. Hence, for normal and nonnormal distributions, the joint default probabilities remain unchanged, whereas the concentration factor can differ substantially. In the case of independence, joint default probabilities of two and three companies are (approximately) zero in the example considered and only individual default occurs within the group. With increasing dependence, the probability of a single default

\footnotetext{
${ }^{3}$ In the case of lognormal $(a, b)$ distribution, the parameters can be calculated by $a=\ln (\mu)-b^{2} / 2$ and $b^{2}=\ln \left(1+\sigma^{2} / \mu^{2}\right)$ (Casella and Berger 2002, p. 109). For gamma distribution $(\alpha, \beta)$, the parameters are given by $\alpha=\mu^{2} / \sigma^{2}$ and $\beta=\sigma^{2} / \mu$ (Casella and Berger 2002, pp. 63-64).

${ }^{4}$ See Aussenegg and Miazhynskaia (2006) or Dockner and Scheicher (1999) for a discussion on different methods on value at risk estimation.
} 


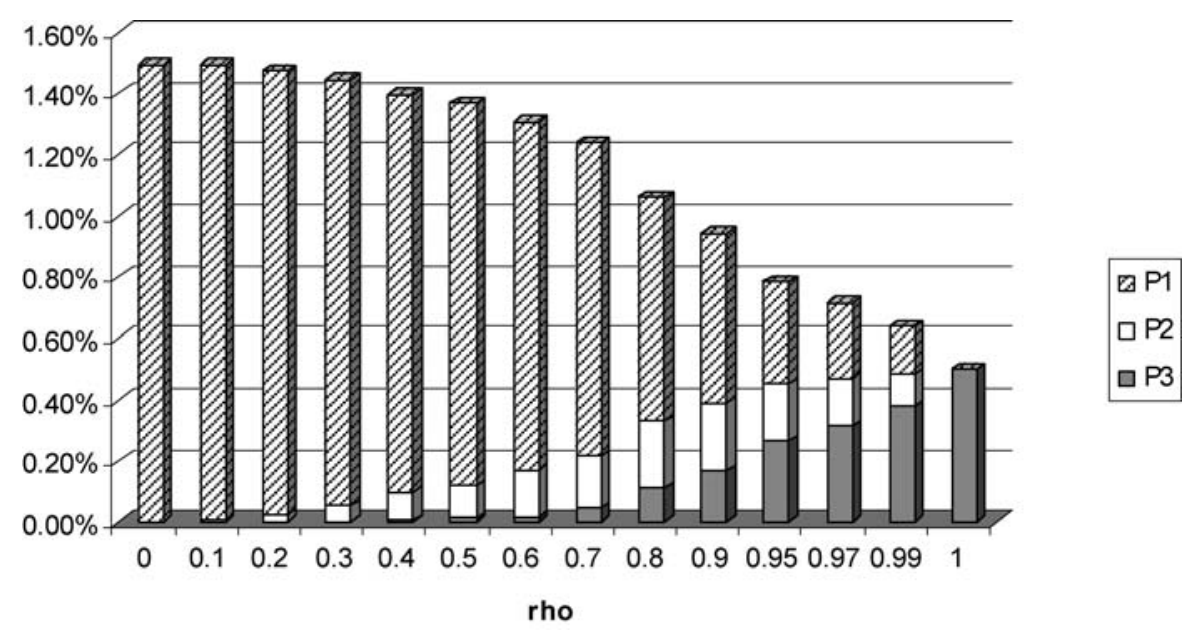

(a) Joint default probabilities for linear dependence

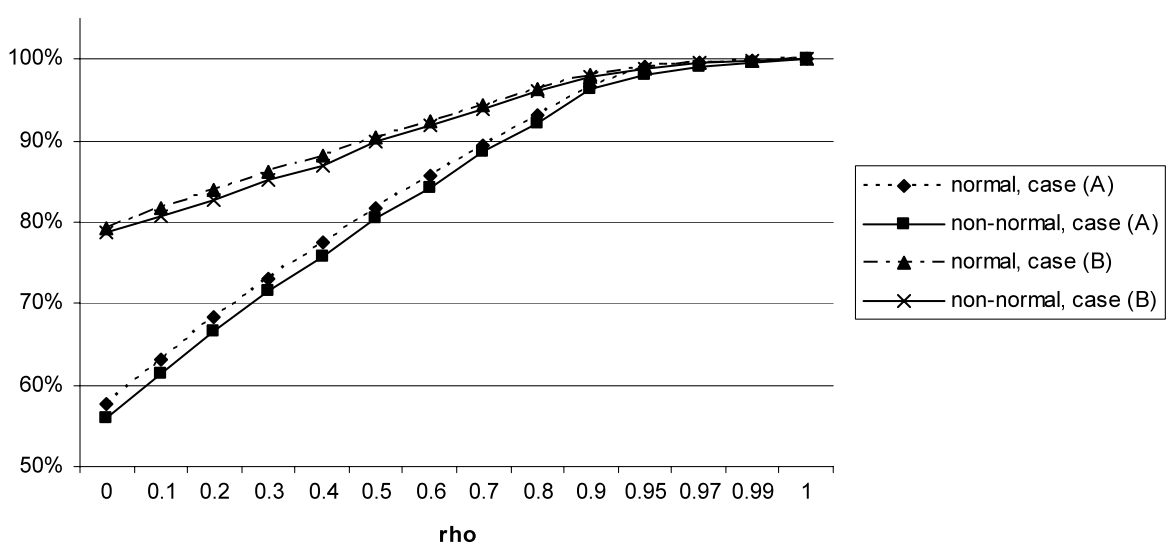

(b) Risk concentration factor for linear dependence

Fig. 1 Default probabilities (in \%) and risk concentration factor (in \%) for linear dependence (rho) on the basis of Table 1 . Notes: $P_{1}=$ probability (in $\%$ ) that exactly one entity defaults; $P_{2}=$ probability (in $\%$ ) that exactly two entities default; $P_{3}=$ probability (in $\%$ ) that all three entities default

$\left(P_{1}\right)$ decreases, while the probability of combined defaults $\left(P_{2}, P_{3}\right)$ increases. For higher correlations, the probability of a combined default of two entities $\left(P_{2}\right)$ decreases again. For perfectly correlated liabilities, all three entities default with probability $0.50 \%$, while $P_{1}=P_{2}=0$.

Part (b) of Fig. 1 illustrates that-given that the liabilities have the same standard deviations (case (A)) - the distributional assumption has only marginal influence on the concentration factor, but that different correlation factors and firm size (case (B)) do matter. As an example, consider the case $\rho=0.3$. The corresponding concentration factor can be derived using (5). For normally distributed liabilities in case (A), the aggregated economic capital is $E C_{a g g r}^{A}=84.65$. This is lower than the sum of stand-alone economic capital (115.91) due to diversification effects. Hence, 
the concentration factor is given by $d=84.65 / 115.91=73.03 \%$. Changing only the distributional assumption to a nonnormal distribution leads to a lower value of $d=90.78 / 126.70=71.65 \%$. The concentration factor decreases in case of nonnormal distribution even though the aggregated economic capital increases to 90.78 . This illustrates that an absolute comparison of aggregated economic capital may be misleading.

The concentration factor is very similar to the results for the normal case, with a difference of only 1.38 percentage points, which can be explained by the calibration of the distributions. We calculated the parameters of the lognormal and gamma distributions using the same values for expected value and standard deviation so as to achieve better comparability between different situations. Hence, we can say that in the example given the choice of a nonnormal distribution has very little impact on concentration factors.

A much larger effect can be observed when comparing case (A) with case (B). For normal distribution, the concentration factor for case (B) is $d=99.76 / 115.91=$ $86.07 \%$. Hence, this situation leads to a higher concentration factor than case (A) $(d=73.03 \%)$. Thus, the situation given in case (B) indicates a possible existence of risk concentration within the group, originating from the bank. The bank's relatively large risk contribution to total group risk causes a less effective diversification of risks. Losses resulting from banking activities in case (B) are less likely to be compensated by good results from insurance activities than in case (A). Thus, the concentration factor $d$ is useful for examining the existence of risk concentrations whenever a benchmark company is available.

Overall, Fig. 1, part (b) demonstrates that the difference between the concentration factors of cases (A) and (B) decreases with increasing correlation. In the case of perfect positive correlation, $\rho=1$, the difference vanishes and the concentration factor takes on its maximum of $100 \%$. Even though all four curves imply the same joint default probabilities, they have different risk concentration factors. The differences in $d$ result from changes in the amount of economic capital needed to retain a constant default probability.

\subsection{Numerical results for nonlinear dependence}

In this section, we alter the assumption for the dependence structure and examine the impact of nonlinear dependencies on risk concentration and joint default probabilities using Clayton and Gumbel copulas as described in the previous section. These dependence structures are tractable and manageable, which is an important prerequisite in practice. Furthermore, the use of the upper tail dependent Gumbel copula can be heuristically motivated by similar strategic decisions in a highly centralized financial group that may lead to a strong degree of tail dependence. Compared to an entity in the same industry that is not part of, and thus not subject to decisions that concern the whole group, these decisions can indeed imply heavy tail dependencies. The lower tail dependent Clayton copula, in contrast, provides a lower bound for risk concentration and default risk, for instance, in the case of a more decentralized group. Both copulas are constructed using Monte Carlo simulation with the same 200,000 paths so as to increase comparability. The Clayton and Gumbel copulas are simulated 


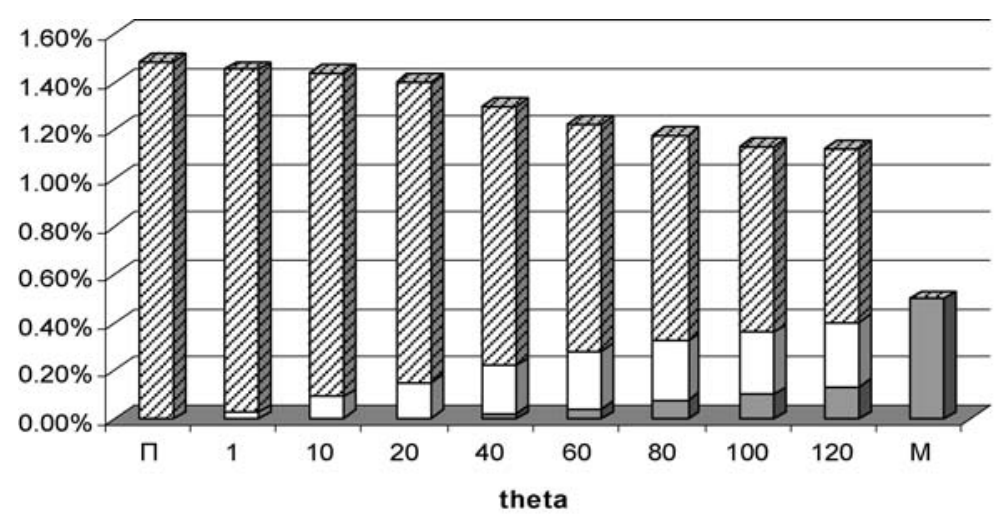

(a) Joint default probabilities for Clayton copula

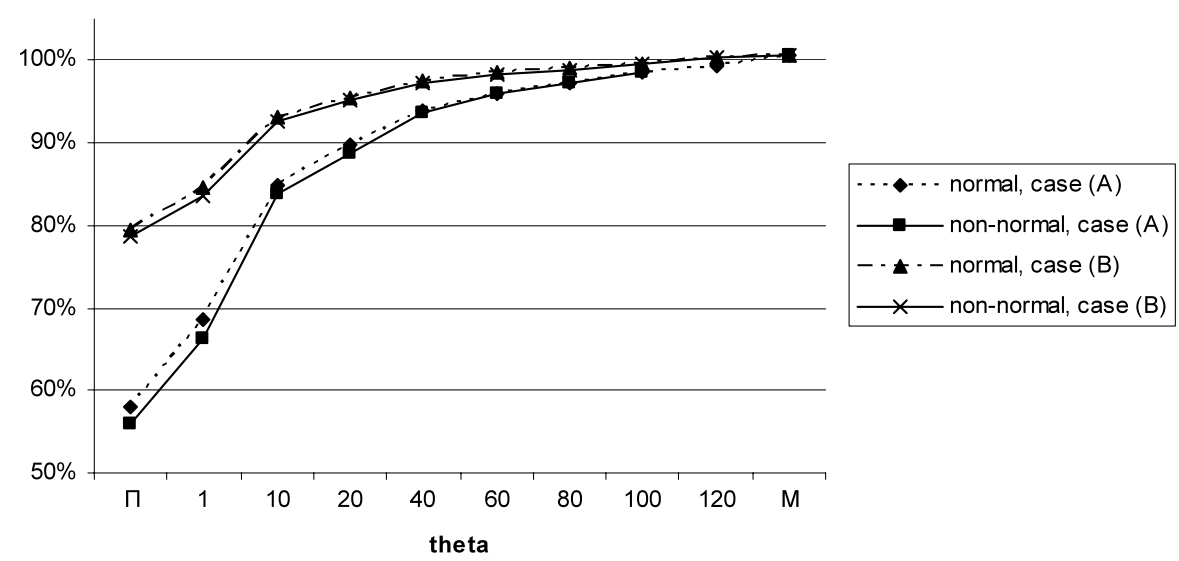

(b) Risk concentration factor for Clayton copula

Fig. 2 Default probabilities (in \%) and risk concentration factor (in \%) for Clayton copula on the basis of Table 1. (Tail dependence parameter: theta.) Notes: $P_{1}=$ probability (in $\%$ ) that exactly one entity defaults; $P_{2}=$ probability (in $\%$ ) that exactly two entities default; $P_{3}=$ probability (in $\%$ ) that all three entities default

using the algorithms in McNeil et al. (2005, p. 224). The algorithm for the Gumbel copula uses positive stable variates, which were generated with a method proposed in Nolan (2005). The estimation of copulas and respective dependence parameters between different market indices from financial market data is described in Aas (2004). A survey on different methods of fitting copula models to empirical data is given in Panjer (2006). Numerical results for the Clayton and Gumbel copulas are illustrated in Figs. 2 and 3, respectively.

In both figures, part (a) displays default probabilities as a function of the dependence parameter $\theta$ and part (b) shows the corresponding concentration factors. ${ }^{5}$ The

\footnotetext{
${ }^{5}$ In the case of the Clayton copula, $0 \leq \theta<\infty$. For $\theta \rightarrow \infty$, one obtains perfect dependence; $\theta \rightarrow 0$ implies independence (McNeil et al. 2005, p. 223). In the case of the Gumbel copula, $\theta \geq 1$; for $\theta \rightarrow \infty$, one obtains perfect dependence, and $\theta \rightarrow 1$ implies independence (McNeil et al. 2005, p. 220).
} 


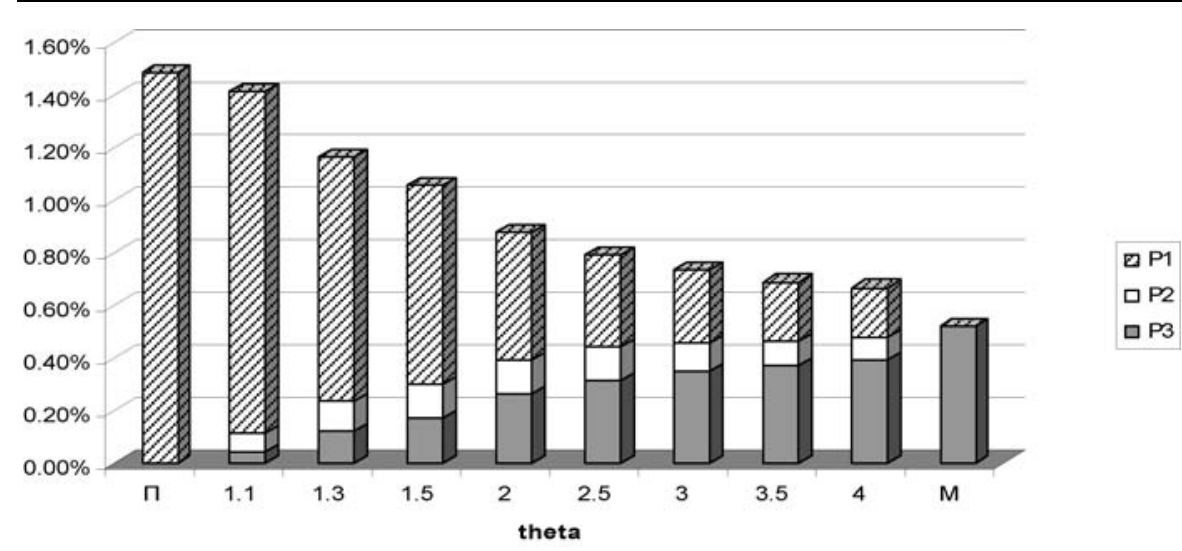

(a) Joint default probabilities for Gumbel copula

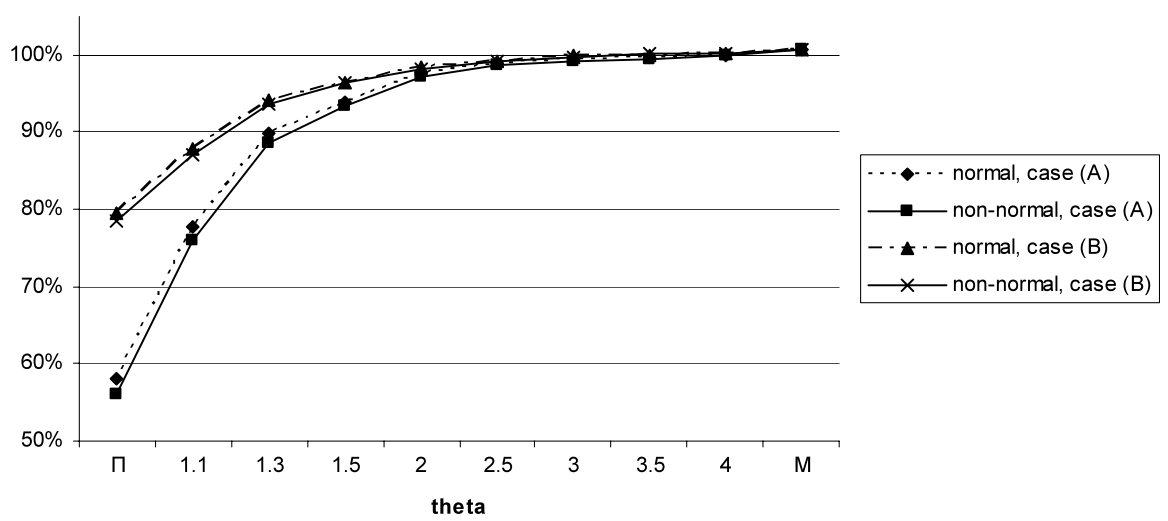

(b) Risk concentration factor for Gumbel copula

Fig. 3 Default probabilities (in \%) and risk concentration factor (in \%) for Gumbel copula on the basis of Table 1. (Tail dependence parameter: theta.) Notes: $P_{1}=$ probability (in $\%$ ) that exactly one entity defaults; $P_{2}=$ probability (in $\%$ ) that exactly two entities default; $P_{3}=$ probability (in $\%$ ) that all three entities default

independence copula marked as $\Pi$ in the figures serves as a lower boundary, while the case of comonotonicity $(M)$ represents perfect dependence and is thus an upper bound.

At first glance, both dependence structures in Figs. 2 and 3 appear to lead to similar results as in the linear case in Fig. 1. Overall, the probability that any company defaults decreases with increasing $\theta$. As before, under perfect comonotonicity, all three entities always become insolvent at the same time with probability $0.50 \%$, while the probability for one or two defaulted companies is zero $\left(P_{1}=P_{2}=0\right)$. In fact, in this case, the concentration factor exceeds $100 \%$ since the value at risk is not a subadditive risk measure (for a discussion, see Embrechts et al. 2002, p. 212).

A comparison of Figs. 2 and 3 reveals that the type of tail dependence (upper vs. lower) has a significant impact on the particular characteristics of the joint default probabilities curves. In case of the upper tail dependent Gumbel copula, companies 
become insolvent far more often, and hence the joint default probability of all three entities quickly approaches $0.50 \%$ in the limit $M$. In contrast, the default probabilities of the lower tail dependent Clayton copula converge to $0.50 \%$ much more slowly. In fact, even for $\theta$ close to 120 , the generation of random numbers from the Clayton copula becomes increasingly difficult, despite the fact that the joint default probability of all three entities is only $0.13 \%$.

Even though results for default probabilities and concentration factors under the Gauss, Gumbel, and Clayton copulas look very similar at first glance, they can differ tremendously, which will be demonstrated in the next subsection.

\subsection{Comparing the impact of nonlinear and linear dependencies}

To compare and identify the considerable effects of the underlying dependence structures on default probabilities, we take examples from the Figs. 1, 2, and 3 that have the same concentration factor, using case (A) with normally distributed marginals so as to make the results comparable.

Two examples are presented in Fig. 4 for fixed concentration factors of $90 \%$ in part (a) and $99.40 \%$ in part (b) from the Clayton, Gauss, and Gumbel copulas. The

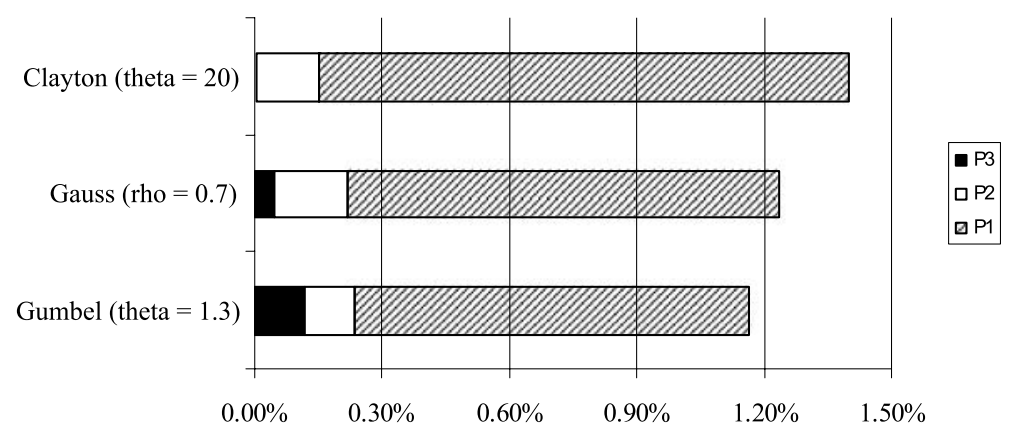

(a) Risk concentration factor $d=90 \%$

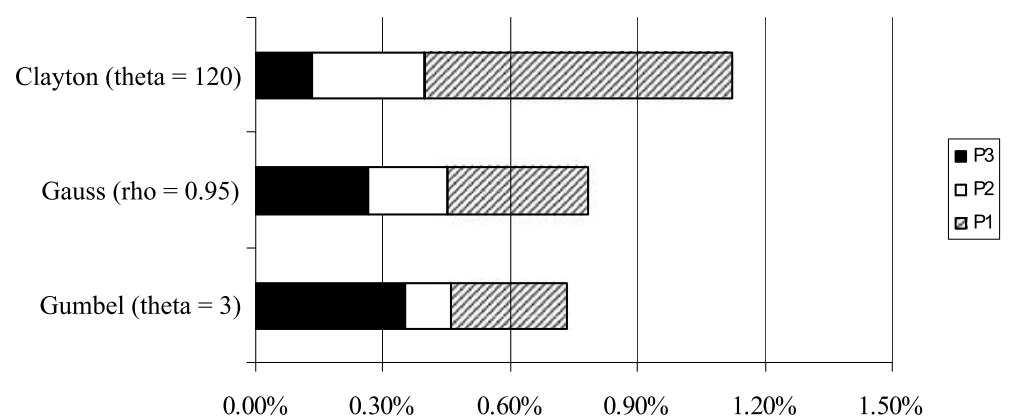

(b) Risk concentration factor $d=99.40 \%$

Fig. 4 Comparison of joint default probabilities (in \%) for one $\left(P_{1}\right)$, two $\left(P_{2}\right)$, and three $\left(P_{3}\right)$ companies for different dependence structures; case (A), normal distributions. Notes: $P_{1}=$ probability (in $\%$ ) that exactly one entity defaults; $P_{2}=$ probability (in $\%$ ) that exactly two entities default; $P_{3}=$ probability (in $\%)$ that all three entities default 
examples in each part of the figure have the same concentration factor and thus exhibit the same value at risk. Although the compared companies have the same risk, default probabilities differ substantially with the dependence structure.

A comparison of parts (a) and (b) of Fig. 4 shows that the sum of default probabilities $\left(=P_{1}+P_{2}+P_{3}\right)$-i.e., the probability that one, two, or three companies default-is higher for the lower concentration factor $d=90 \%$. Furthermore, for $d=99.40 \%$, the partitioning between the three joint default probabilities $\left(P_{1}, P_{2}, P_{3}\right)$ is shifted toward $P_{3}$, while $P_{1}$ decreases. Hence, a higher concentration factor is accompanied by a lower sum of default probabilities, but induces a significantly higher joint default probability of all three entities.

Figure 4 demonstrates the considerable influence that the choice between Clayton, Gauss, and Gumbel copulas has on joint default probabilities. The Clayton copula leads to the highest sum of default probabilities, but has the lowest probability of default for all three companies $\left(P_{3}\right)$. The other extreme occurs under the Gumbel copula, where $P_{3}$ is highest and $P_{1}$ takes the lowest value, while the Gauss copula induces values between those of the Clayton and Gumbel copulas.

Our results show that even if different dependence structures imply the same value at risk, and thus the same risk concentration factor, joint default probabilities can differ tremendously. These findings strongly depend on the underlying assumption regarding the portfolio of firms. First, concentration factors can be calculated only under the theoretical assumption that risks of all separate entities in the group are merged into one single groupwide portfolio, allowing for a full transfer of losses. In the case of no loss transfers, the analysis of default probabilities is generally valid for any type of portfolio the entities have. Second, the specific group structure (centralized, decentralized) plays an immense role for the dependence structure and thus for concentration and joint default risk.

Our analysis demonstrates that the simultaneous reporting of risk concentration factors and default probabilities can be of substantial value, especially for the management of the corporate group. By comparing linear and nonlinear dependencies, we found that the effect of mismodeling dependencies may not only lead to significant differences in assessing risk concentration, but can also lead to misestimating joint default probabilities. Hence, there is a substantial model risk involved with respect to dependence structures.

\section{Conclusion}

This paper assessed and related risk concentrations and joint default probabilities of legal entities in a group. Our procedure provided valuable insight regarding the group's risk situation, which is highly relevant for enterprise risk management purposes. A financial group typically consists of several legally independent entities, each with limited liability. However, diversification concepts assume that these entities are fully liable and all together meet all outstanding liabilities of each. Even if diversification is of no importance from a policyholder perspective, it is useful in determining risk concentration in a financial group because greater diversification generally implies less risk. 
To determine default probabilities, we focused on the case of limited liability without transfer of losses within the group. In the numerical analysis, we considered a financial group comprised of three legal entities and compared results from the Gauss, Gumbel, and Clayton copulas for normal and non-normal marginal distributions. Economic capital was adjusted for each situation to satisfy a fixed individual default probability. In contrast to the risk concentration factor, joint default probabilities only depend on individual default probabilities and on the dependence structure, not on distributional assumptions. Hence, we studied the effect of different dependence structures using the concept of copulas.

For all models, we found that the risk concentration factor and the joint default probability of all three entities increase with increasing dependence between the entities, while the probability of a single default decreases. Overall, the sum of default probabilities of one, two, or three entities decreases with increasing dependence. Furthermore, one entity's large risk contribution led to a much higher risk concentration factor for the group as a whole. Our findings further demonstrated that even if different dependence structures imply the same risk concentration factor for the group, joint default probabilities for different sets of subsidiaries can vary tremendously.

The analysis showed that a simultaneous consideration of risk concentration factors and default probabilities can be of substantial value, especially for the management of a financial group with respect to enterprise risk management. In particular, information about joint default probabilities is of interest if there is no loss compensation between entities in a group. Risk concentration can be approximated by assuming that losses are fully offset, even though, in reality, legal restrictions might prevent this.

In practice, several issues should be considered in the process of implementation. Our results demonstrated that it is not only distributional assumptions that are relevant, but that the dependence structure between different entities of a group is particularly important. In this respect, consideration and estimation of nonlinear dependencies is vital and a sensitivity analysis of default probabilities and risk concentration allows an assessment of the model risk with respect to misjudged dependencies and distribution.

Acknowledgements The authors wish to thank two anonymous referees for helpful comments on an earlier draft of this paper. Financial support from the Swiss National Science Foundation (SNF) is grateful acknowledged.

\section{References}

Aas, K.: Modelling the dependence structure of financial assets: a survey of four copulas. Research Report SAMBA/22/04, Norwegian Computing Center (2004)

Amel, D., Barnes, C., Panetta, F., Salleo, C.: Consolidation and efficiency in the financial sector: a review of the international evidence. J. Bank. Finance 28(10), 2493-2519 (2004)

Ammann, M., Reich, C.: VaR for nonlinear financial instruments-linear approximation or full Monte Carlo. Financ. Mark. Portf. Manag. 15(3), 363-378 (2001)

Aussenegg, W., Miazhynskaia, T.: Uncertainty in value-at-risk estimates under parametric and nonparametric modeling. Financ. Mark. Portf. Manag. 20(3), 243-264 (2006)

Bikker, J.A., Lelyveld, I.P.P.: Economic versus regulatory capital for financial conglomerates. Research Series Supervision No. 45, Netherlands Central Bank (2002) 
CAS: Overview of Enterprise Risk Management (2003). Available at http://www.casact.org/research/erm/ Casella, G., Berger, R.L.: (2002): Statistical Inference. Duxbury Press, Pacific Grove

CEIOPS: Advice to the European Commission in the Framework of the Solvency II Project on Sub-Group Supervision, Diversification Effects, Cooperation with Third Countries and Issues Related to the MCR and SCR in a Group Context (CEIOPS-CP-05/06) (2006). Available at http://www.ceiops.org/content/view/14/18

CEIOPS: QIS3-Guidance on the QIS3 package (CEIOPS-FS-20/07) (2007). Available at http://www.ceiops.org/content/view/118/124

Daykin, C.D., Pentikainen, T., Pesonen, M.: Practical Risk Theory for Actuaries. Chapman \& Hall, London (1994)

Dimakos, X.K., Aas, K.: Integrated risk modelling. Stat. Model. 4(4), 265-277 (2004)

Dockner, E., Scheicher, M.: Evaluating volatility forecasts and empirical distributions in Value at Risk models. Financ. Mark. Portf. Manag. 13(1), 39-55 (1999)

Embrechts, P., McNeil, A., Straumann, D.: Correlation and dependence in risk management: properties and pitfalls. In: Dempster, M.A.H. (ed.) Risk Management: Value at Risk and Beyond, pp. 176-223. Cambridge University Press, Cambridge (2002)

Embrechts, P., Lindskog, F., McNeil, A.J.: Modelling dependence with copulas and applications to risk management. In: Rachev, S. (ed.) Handbook of Heavy Tailed Finance, pp. 329-384. Elsevier, Amsterdam (2003)

Estrella, A.: Mixing and matching: prospective financial sector mergers and market valuation. J. Bank. Finance 25(12), 2367-2392 (2001)

European Commission: Policy Issues for Solvency II-Possible Amendments to the Framework for Consultation (Markt/2505/01). Brussels (2005)

European Commission: Proposal for a Directive of the European Parliament and of the Council on the Taking up and Pursuit and Business of Insurance and Reinsurance, Solvency II (COM(2007) 361 final). Brussels (2007)

European Council: Directive 98/78/EC of the European Parliament and of the Council of 27 October 1998 on the supplementary supervision of insurance undertakings in an insurance group. Official J. Eur. Union L330, 1-12 (1998)

European Council: Directive 2002/87/EC of the European Parliament and of the Council of 16 December 2002 on the supplementary supervision of credit institutions, insurance undertakings and amending Council Directives 73/239/EEC, 79/267/EEC, 92/49/EEC, 92/96/EEC, 93/6/EEC and 93/22/EEC, and Directives 98/78/EC and 2000/12/EC of the European Parliament and of the Council. Official J. Eur. Union L35, 1-26 (2002)

Faivre, F.: Copula: a new vision for economic capital and application to a four line of business company. Paper presented at ASTIN Colloquium, Berlin (2003)

Frees, E.W., Valdez, E.V.: Understanding relationships using copulas. N. Am. Actuar. J. 2(1), 1-25 (1998)

Frey, R., McNeil, A.J.: Modelling Dependent Defaults. ETH E-Collection, Zürich (2001). Available at http://e-collection.ethbib.ethz.ch

Groupe Consultatif: Diversification-Technical Paper. Working Group "Group and Cross-Sectoral Consistency” (2005). Available at http://www.gcactuaries.org/solvency.html

Hull, J.C.: Options, Futures and Other Derivatives. Pearson Education, Upper Saddle River (2003)

Iman, R.L., Conover, W.: A distribution free approach to inducing rank correlation among input variables. Commun. Stat. Simul. Comput. 11(3), 311-334 (1982)

Kuritzkes, A., Schuermann, T., Weiner, S.M.: Risk measurement, risk management and capital adequacy in financial conglomerates. In: Brookings-Wharton Papers on Financial Services, pp. 141-193 (2003)

Li, D.X.: On default correlation: a copula function approach. J. Fixed Income 9(4), 43-54 (2000)

McNeil, A.J., Frey, R., Embrechts, P.: Quantitative Risk Management. Concepts, Techniques, Tools. Princeton University Press, Princeton (2005)

Nelsen, R.B.: An Introduction to Copulas. Springer, New York (1999)

Nolan, J.P.: Stable distributions: models for heavy tailed data (2005, forthcoming)

Panjer, H.H.: Operational Risk Modelling Analytics. Wiley, Hoboken (2006)

Rosenberg, J., Schuermann, T.: A general approach to integrated risk management with skewed, fat-tailed risks. J. Financ. Econ. 79(3), 569-614 (2006)

Tang, A., Valdez, E.A.: Economic capital and the aggregation of risks using copula. Paper presented at the 28th International Congress of Actuaries, Paris (2006). Available at http://papers.ica2006.com

Wang, S.: Aggregation of correlated risk portfolios: models and algorithms. Proc. Casualty Actuar. Soc. 85, 848-939 (1998) 
Wang, S.: A set of new methods and tools for enterprise risk capital management and portfolio optimization. In: The Casualty Actuarial Society Forum, Summer 2002, Dynamic Financial Analysis Discussion Papers, pp. 43-77 (2002)

Ward, L.S., Lee, D.H.: Practical application of the risk adjusted return on capital framework. In: The Casualty Actuarial Society Forum, Summer 2002, Dynamic Financial Analysis Discussion Papers, pp. 79-126 (2002)

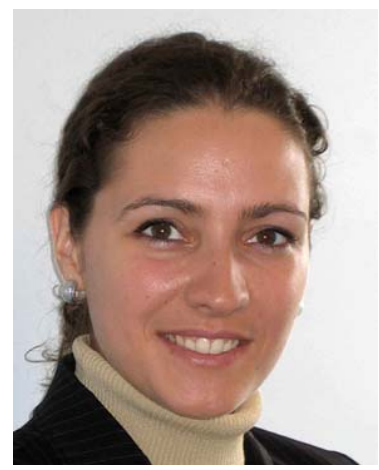

Nadine Gatzert studied mathematics and economics at the University of Ulm, Germany, and at the University of Southern California in Los Angeles, USA, majoring in mathematical finance and actuarial science. Since 2005, she is a research assistant and project manager at the Institute of Insurance Economics of the University of St. Gallen, Switzerland. After receiving her doctoral degree in 2007, she currently holds a postdoctoral position. Her research interests include asset-liabilitymanagement, alternative risk transfer, and embedded options in life insurance contracts.

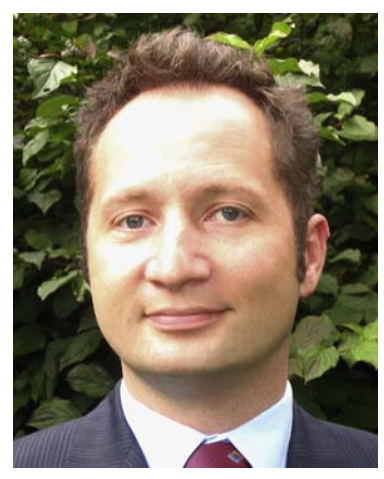

Hato Schmeiser studied business administration at the University of Manheim, Germany. After his doctoral degree and the postdoctoral lecture qualification (Habilitation) in 2003 (Humboldt-Universität zu Berlin), he was appointed Professor for Insurance and Risk Management at the University of Münster. Since 2005, he is Chair for Risk Management and Insurance at the University of St. Gallen. His main research interests include individual financial planning, dynamic financial analysis, option pricing, and regulation of financial firms.

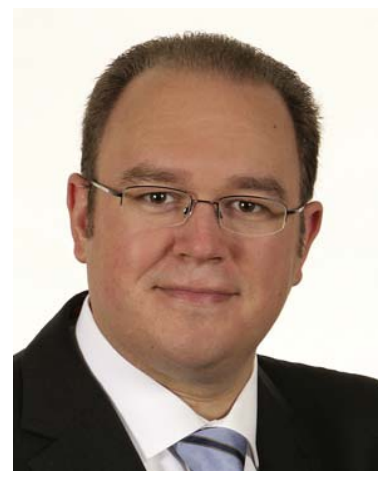

Stefan Schuckmann studied business administration at the University of Muenster, Germany, and at the University of Vienna, Austria, majoring in risk and insurance management. From 1999 to 2005 he worked for Deutsche Bank's Private and Business Clients division. Since then, he is research assistant and project manager at the Institute of Insurance Economics at the University of St. Gallen, Switzerland. His main research interests include value based management, asset liability management, and regulation of financial firms. 